\title{
Ab initio calculations of elastic properties of alloys with mechanical instability: Application to BCC Ti-V alloys
}

Natalia Skripnyak, A. V. Ponomareva, M. P. Belov and Igor Abrikosov

The self-archived postprint version of this journal article is available at Linköping University Institutional Repository (DiVA):

http:/ / urn.kb.se/ resolve?urn=urn:nbn:se:liu:diva- 145445

N.B.: When citing this work, cite the original publication.

Skripnyak, N., Ponomareva, A. V., Belov, M. P., Abrikosov, I., (2018), Ab initio calculations of elastic properties of alloys with mechanical instability: Application to BCC Ti-V alloys, Materials \& design, 140, 357-365. https:// doi.org/ 10.1016/j.matdes.2017.11.071

Original publication available at:

https:// doi.org/ 10.1016/j.matdes.2017.11.071

Copyright: Elsevier

http:// www.elsevier.com/

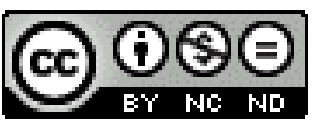




\title{
AB INITIO CALCULATIONS OF ELASTIC PROPERTIES OF ALLOYS WITH MECHANICAL INSTABILITY: APPLICATION TO BCC Ti-V alloys.
}

\author{
N. V. Skripnyak ${ }^{1}$, A. V. Ponomareva², M. P. Belov², I. A. Abrikosov ${ }^{1, *}$ \\ ${ }^{1}$ Department of Physics, Chemistry and Biology, Linköping University, Linköping, SE-581 83, Sweden \\ ${ }^{2}$ Materials Modeling and Development Laboratory, National University of Science and Technology "MISIS", \\ Moscow, 119049, Russia \\ *Corresponding author: \\ Address: Department of Physics, Chemistry and Biology, Linköping University, Linköping, SE-581 83, \\ Sweden; \\ E-mail address: igor.abrikosov@ifm.liu.se
}

\begin{abstract}
Considering Ti-V alloys with the body-centered cubic crystal lattice, a system with mechanical instability for Ti-rich alloys, we calculate their elastic properties using Projector Augmented Wave method and the exact muffin-tin orbital method in a complete interval of $\mathrm{V}$ concentrations. The substitutional disorder is modeled using the special quasi-random structures technique and the coherent potential approximation. The efficiency and accuracy of the simulation techniques is analyzed, and a strategy for efficient high-throughput calculations of elastic properties of disordered alloys is proposed. Dependences of the single crystal elastic moduli on V concentration and a set of mechanical characteristics of polycrystalline alloys are presented and discussed. The effect of $\mathrm{V}$ content on the mechanical stabilization of the bcc Ti-V alloys is investigated. In agreement with experiment, we find that titanium-rich alloys are mechanically unstable, however the alloys become mechanically stable with increasing content of $\mathrm{V}$ in the system. We observe a nonlinear dependence of the alloys Young's moduli in a vicinity of the mechanical stabilization and suggest that this effect can be used to design alloys with low values of the elastic moduli.
\end{abstract}

Keywords: efficient first-principal simulations, elastic moduli, substitutional disorder, Ti-V alloys, mechanical stabilization

\section{Introduction}

An important challenge related to a data-driven development of new materials is to assemble large databases of the physical, chemical and structural properties of the phases. A use of the databases and the generated knowledge in combination with improved machine learning techniques should significantly reduce the costs for the development of new functional materials and shorten their development time. There are different approaches to generate data on elastic, thermochemical, thermodynamic and structural properties in a wide range of external conditions. First-principles modeling, semi-empirical and empirical methods can be applied, and a work focused on obtaining data on the structure and properties of the phases of various materials by machine learning methods has been initiated [1]. Unfortunately, materials science databases are 
usually much smaller than those that are available in other areas, where machine learning has already proven its usefulness. Insufficient large sample can lead to over-fitting of model and / or poor predictive accuracy of machine learning methods. Moreover, materials science databases obtained from the first principles contain information primarily about ordered structures and their extension to alloys is an important scientific task on its own, as well as for the development of machine learning and data mining techniques within materials science.

The required expansion leads to the need to deal with a configurational disorder present in solid solutions. A thoughtful review of the challenging theoretical task in terms of simulations of the alloy thermodynamics is presented in Ref. [2]. The most straight-forward approach consists of employing the so-called supercells, e.g. using the Special Quasirandom Structures (SQS) method [3]. Consequently, the treatment of the disordered material is technically the same as in the standard electronic structure calculations of ordered compounds, e.g. at the same level of accuracy. In particular, the full non-spherical shape of the crystal potential can be treated explicitly, which makes it possible to carry out the full relaxation of atomic positions in the supercell. Unfortunately, the supercells often contain several dozens of atoms, which greatly (as $N^{3}$, where $N$ is number of atoms in the SQS) increases the costs of such calculations in comparison to those for ordered compounds. This makes the use of the SQS technique less practical in high-throughput calculations needed for the construction of the data-bases. In this regard, the use of numerically more efficient, though probably more approximate methods becomes important [1]. However, the reliability of properties predicted by those methods must be established in advance.

In particular, the use of the exact muffin-tin orbital (EMTO) method combined with the coherent potential approximation (CPA) appears to be promising [4]. The technique allows for very efficient, with $O\left(N^{0}\right)$ rather than $O\left(N^{3}\right)$ scaling of the electronic structure calculations needed for the description of thermodynamic and elastic properties of alloys $[2,4,5]$. Unfortunately, the electronic structure problem within this method is solved using the spherical approximation for the one-electron potential. Moreover, the CPA is a single-site mean-field approximation. Because of these limitations, the EMTO-CPA technique does not allow for atomic relaxations and it is not a truly full-potential technique. Therefore, the reliability of the EMTO-CPA calculations must be verified in comparison with more accurate calculations. In this paper, a detailed comparison of elastic moduli calculated by means of the supercell technique with the Projector Augmented Wave method [6] for a set of SQS (PAW-SQS) and by means of the EMTO-CPA method is carried out. As a model system, we have chosen bcc Ti-V alloys.

The Ti-V alloys are of practical interest for high-temperature structural elements of the aerospace and automotive industries, as well as for the development of nuclear reactors. Vanadium is one of the so-called $\beta$-stabilizers of titanium alloys. That is, the alloying with vanadium makes it possible to stabilize the high-temperature bcc phase of titanium at temperatures below $1155 \mathrm{~K}$, which is often beneficial for the mechanical properties of titanium alloys in structural elements operating in a wide range of temperatures. Another advantage of vanadium as an alloying element in titanium alloys is the absence of eutectoid reactions and intermetallic phases in the Ti-V system. This almost eliminates the appearance of brittleness in cases of errors in carrying out technological processes associated with heating. In addition, Ti-V alloys have a very narrow range of crystallization [7-9]. Moreover, it is known that vanadiumrich alloys with Ti additions combine low temperature strength and high ductility with high strength at elevated temperature and low creep [10]. An interest in the properties of $\beta$-titanium alloys has further increased in view of their application to create elements of designs by using 
Selective Laser Sintering (SLS) technology [11]. An applicability of Ti-V alloys for hydrogen storage has been considered as well [12-13].

Elastic moduli are important characteristics of structural materials and are used to evaluate their strength and stability. In this respect, bcc titanium alloys attract attention as alloys having a low Young's modulus [14-15]. For this study, it is important to underline that bcc Ti is mechanically unstable at temperature $\mathrm{T}=0 \mathrm{~K}$, that is its elastic moduli $C^{\prime}$ is negative. It is therefore important to establish how different computational methods deal with the instability in simulations of elastic properties of bcc Ti alloys. The elastic moduli of Ti-V system have been studied in several experimental [16-18] and theoretical works [19-21]. In Ref. [20] the elastic constant $C^{\prime}$ was calculated in the Ti-rich region within the virtual crystal approximation, and the mechanical instability of the bcc alloy in this region was indeed observed. In Ref. [21] V- rich region was examined by the EMTO method and all the elastic constants of the crystal were calculated. In Ref. [19], using the ultra-soft pseudopotentials, the elastic properties of several ordered Ti-V compounds of different compositions in the intermediate concentration range were calculated, and the conclusions have been drawn on the behavior of the elastic properties of disordered alloys. At the same time, in the comparison of the energies of ordered and random TiV structures [22], it was shown that SQS supercells used to model the random alloys have substantially lower energy, so the calculations of the properties of ordered compounds in the TiV system may not describe the real disordered solution phase. Importantly, the earlier results are not complete because they do not cover the entire range of concentration of the alloy.

The aim of this work consists in a systematic study the of the elastic properties of disordered bcc Ti-V alloys in the entire concentration range by using the PAW + SQS and EMTO-CPA methods, and carrying out detailed comparative analysis of the accuracy and the efficiency of the calculations of elastic properties with these two techniques. Specifically, we are interested whether numerically more efficient EMTO-CPA method is suitable for a rapid search for trends of the materials elastic properties and for providing data for materials science databases in the case of multicomponent disordered solid solutions with mechanical instability.

\section{Methods}

The first-principles modeling of Ti-V systems was carried out by using the PAW method [6] implemented in the Vienna Ab-Initio Simulation Package (VASP) [23-24] and the Exact Muffin-Tin Orbitals (EMTO) method in the coherent potential approximation [4]. The exchangecorrelation effects in an electron gas were taken into account within the density functional theory in the framework of the generalized gradient approximation (GGA) [25].

\subsection{Details of EMTO-CPA calculations}

In the EMTO method, the total (non-spherically symmetric) charge density is obtained by an exact self-consistent solution of the one-electron Kohn-Shem equations for overlapping spherical Muffin-Tin (MT) potentials. This makes it possible to achieve accuracy comparable to full-potential methods, while retaining the effectiveness of methods based on the use of the MT potentials. The combination of the EMTO method with the CPA, presented in Ref. [4] makes it possible to carry out calculations for disordered substitutional alloys. In the framework of the 
CPA, the latter is approximated by an effective medium with the same number of atoms $N$ as in the corresponding ordered system, making the scaling of simulations $\mathrm{O}\left(N^{0}\right)$ rather than $\mathrm{O}\left(N^{3}\right)$ as in supercell calculations. The effective medium is constructed in such a way that the electron scattering off the effective atoms is the same, on the average, as in the simulated disordered alloy. This condition is ensured by a self-consistent solution of a system of CPA equations formulated in terms of the Green's function technique [2].

To describe an alloy with the body-centered cubic (bcc) crystal lattice within the CPA $N=1$. This makes the simulations very efficient, and allows one to use calculation parameters that guaranteed highly converged results. Indeed, even with the parameters specified below the total computer time for calculations of the elastic moduli at 13 different concentrations is just 1300 core/hours on Intel Xeon E5-2650 processors. The disadvantages of this method are the absence of local relaxations and the use of the spherical MT potential.

The calculation parameters were as follows. The basic set of EMTO included s-, p-, dand f - orbitals. The full charge density (FCD) [26] was represented by a single-center expansion of the electron wave functions in terms of spherical harmonics with orbital angular moments $l_{F C D}^{\max }=8$. The integration in the irreducible part of the Brillouin zone was performed over a 29x29x29 grid of k points. The energy integration was carried out in the complex plane using a semielliptic contour comprising 24 energy points. The convergence of energy with respect to the calculation parameters was $10^{-8} \mathrm{Ry}$.

In order to determine the elastic constants $C^{\prime}$ and $C_{44}$ of $\mathrm{Ti}-\mathrm{V}$ alloys we applied volumeconserving orthorhombic (1) and monoclinic (2) distortions, respectively, and calculated the internal energy response to the small distortions:

$$
\begin{aligned}
& 1+\varepsilon_{1}(\eta)=\left(\begin{array}{ccc}
1+\eta & 0 & 0 \\
0 & 1-\eta & 0 \\
0 & 0 & \frac{1}{1-\eta^{2}}
\end{array}\right) \\
& 1+\varepsilon_{2}(\eta)=\left(\begin{array}{ccc}
1 & \eta & 0 \\
\eta & 1 & 0 \\
0 & 0 & \frac{1}{1-\eta^{2}}
\end{array}\right)
\end{aligned}
$$

The total energy was calculated for six distortions $(\eta=0.00-0.05)$.

\subsection{Calculation details of the PAW-SQS method}

The PAW method [6] was used within the framework of the density functional theory, as it implemented in the VASP software package [23-24]. The disorder problem was treated within the SQS approach [3]. It allows one to perform simulations of the disordered alloy modelled by a quasi-random, but still ordered supercell, enabling the straight-forward use of the PAW method. The SQS are constructed in such a way as to ensure that the short-range order parameters for the several neighboring coordination shells in the supercell are as close to zero on the average as possible, similar to their values in the case of complete disorder [2]. The SQS for bcc Ti-V alloys was constructed for a 128-atom supercell consisting of $4 \times 4 \times 4$ simple cubic cells. To determine the numerical parameters of elastic constant calculations, a number of tests were performed as discussed in Supporting information. 
To achieve accurate stresses, the cutoff energy had to be set to $460 \mathrm{eV}$. The integration of the Brillouin zone was performed using a set of $3 \times 3 \times 3$ k-points. PAW-SQS calculations with this set of parameters are time-consuming. Even without taking into account the convergence tests, the total computer time to calculate elastic moduli of bcc Ti-V alloys at 7 different concentrations using the PAW - SQS method can be estimated as 67200 core/hours on Intel Xeon E5-2650 processors, or about 2 orders of magnitude more than EMTO-CPA calculations.

To improve the efficiency of PAW-SQS calculations, we used the deformation matrix:

$$
1+\varepsilon_{1}(\eta)=\left(\begin{array}{ccc}
1+\eta & \frac{\eta}{2} & 0 \\
\frac{\eta}{2} & 1 & 0 \\
0 & 0 & 1
\end{array}\right)
$$

which allows us to calculate directly all the necessary moduli [27]. In Eq. (3) $\eta$ is the magnitude of the deformation, which we varied from -0.02 to 0.02 in steps of 0.01 . Accordingly, elastic constants are determined from the following stress-strain relationships:

$$
\mathrm{C}_{11}=\frac{\partial \sigma_{x x}}{\partial \eta}, \quad \mathrm{C}_{12}=\frac{\partial \sigma_{y y}}{\partial \eta}, \quad \mathrm{C}_{44}=\frac{\partial \sigma_{x y}}{\partial \eta},
$$

We note that calculations of the tensorial quantities (like elastic moduli) by a supercell technique is less straight-forward than for the scalar quantities (like total energies). Indeed, the SQS approach does not aim to generate structures that preserve the point group symmetry of an alloy and thus to provide the proper description of its tensorial properties. In almost all practical cases, the use of the SQS method leads to a disordered alloy models, which do not have the full cubic symmetry [28]. Therefore, we employ the projection technique introduced by Moakher and Norris [29] to derive the closest elastic tensors with cubic symmetry in the modeling of the bcc Ti-V alloy. In case of the SQS description of an alloy with a cubic symmetry, it reduces to performing calculations of $C_{11}, C_{12}$, and $C_{44}$ with Eq. (3) for all permutations of the a, b, and c axis [28]. The resulting $C_{11}, C_{12}$, and $C_{44}$ for the alloy are then obtained as the averages of those calculated from Eq. (4) for every permutation of the axis. This procedure ensures the correct symmetry of the elastic moduli and faster convergence of their calculations with the size of the SQS.

Considering the numerical test presented in Supporting information, we observed that conducting the procedure of ionic relaxations does not affect the calculated elastic constants. The observation is important for the use of the EMTO-CPA method which neglects the effect of the local lattice relaxations.

\subsection{Analysis of mechanical stability}

To determine the mechanical stability of the bcc alloys, we used the mechanical stability criterion:

$$
\mathrm{C}^{\prime}>0 \quad(5)
$$

where $\mathrm{C}^{\prime}$ is defined as: 


$$
\mathrm{C}^{\prime}=\frac{1}{2}\left(\mathrm{C}_{11}-\mathrm{C}_{12}\right)
$$

\subsection{Elastic moduli of polycrystalline alloys}

The elastic moduli obtained by the EMTO-CPA and PAW-SQS methods are calculated assuming that the alloy is a single crystal. One of the most widely used methods to estimate the elastic characteristics of polycrystalline materials is the averaging of the single crystal elastic moduli, known as the Voigt (V) -Reuss (R) -Hill (H) procedure [30-32]. Voigt averaged the elastic moduli over all orientations of the lattice, assuming the uniformity of the deformation along the volume of the crystal. On the contrary, Reuss assumed that the stresses are uniform. Hill showed that the Voigt approximation leads to overestimated values of the elastic moduli, while the Reuss approximation to underestimated values, and suggested to use the average of these two approximations [30-32]. The procedure used here is described in [33-34].

The bulk modulus in the Hill approximation $B_{H}$ is determined from the relation:

$$
B_{H}=\frac{B_{R}+B_{v}}{2}
$$

However, for cubic crystals $B_{R}$ and $B_{V}$ are equal:

$$
B_{R}=B_{V}=\frac{\left(C_{11}+2 C_{12}\right)}{3}
$$

The shear modulus in the Hill approximation $G_{H}$ was calculated by analogy from the relation:

$$
G_{H}=\frac{G_{R}+G_{v}}{2}
$$

Where $G_{R}$ is the shear modulus in the Reuss approximation, $G v$ is the shear modulus in the Voigt approximation, which can be written for cubic crystals as:

$$
\begin{array}{r}
G_{R}=\frac{5 C_{44}\left(C_{11}-C_{12}\right)}{4 C_{44}+3\left(C_{11}-C_{12}\right)} \\
G_{v}=\frac{C_{11}-C_{12}+3 C_{44}}{5}
\end{array}
$$

Knowing $B$ and $G$, we calculate the Young's modulus $E$ and the Poisson's ratio $v$ from the relations:

$$
\begin{gathered}
E=\frac{9 B G}{3 B+G} \\
v=\frac{3 B-2 G}{2(3 B+G)}
\end{gathered}
$$


The phenomenological criteria to determine whether a material is expected to be brittle or ductile using the Cauchy pressure $P_{c}$ [35] and the Pugh ratio $k$ [36] are used quite often in practice. They are calculated as:

$$
\begin{gathered}
P_{C}=\left(\mathrm{C}_{12}-\mathrm{C}_{44}\right) \\
k=\frac{G}{B}
\end{gathered}
$$

If $k>0.5$ and $P_{c}<0$, the material is considered to have predominantly covalent character of the chemical bonds and is characterized theoretically as a brittle material [4, 5, 25-26, 35-37].

\section{Results and discussion}

Using the EMTO-CPA and PAW-SQS methods, the dependences of single-crystal elastic constants and the polycrystalline elastic moduli for Ti-V alloys on the concentration of vanadium in the range from 0 to 100 at.\% V were found. In Fig.1 the calculated dependences of the elastic constants $C_{11}, C_{12}, C_{44}$ are displayed. The modules $C_{11}$ and $C_{12}$ obtained by the PAW-SQS and EMTO-CPA methods for the bcc Ti-V alloys increase monotonously with increasing vanadium concentration in the range from 0 to $100 \%$. The experimental data are presented in the range from 30-100 at. \% V and the increase is almost linear in the whole interval of $\mathrm{V}$ concentrations. The obtained PAW-SQS results for $C_{11}$ show good quantitative agreement with the experimental data, as well as good agreement with the data obtained on the ordered structure [19]. EMTO-CPA calculations predict somewhat higher values of $C_{11}$. At the same time, the concentration dependence of $C_{11}$ obtained in EMTO-CPA calculations is linear and it is in somewhat better agreement with experimental concentration dependence than the one predicted by PAW- SQS calculations. One reason for the kinks that we see in PAW-SQS calculations of $C_{11}$ could be incomplete k-point convergence. Indeed, it is much easier to fully converge the EMTO-CPA calculations, as they are done for one (effective) atom per cell rather than PAW- SQS calculations for a supercell with 128 atoms (Supporting information). In this sense, the efficiency of the EMTO-CPA method turns out to be beneficial for the accuracy of the trends, predicted with this method, even though the absolute values obtained with PAW-SQS calculations are in better agreement with experiment. Simultaneously, we see very good agreement between $C_{12}$ elastic modulus obtained by the PAW-SQS and EMTO-CPA methods. In this case, the agreement with experiment is similar for the two methods. Again, the latter predicts a smoother concentration dependence than the former, most probably because of the better numerical convergence. In summary, the results of the calculations obtained by the PAW-SQS and EMTO-CPA methods correctly reproduce the trends and are in reasonable agreement with each other and with experimental data [16,17]. The largest deviation is observed in the vanadium-rich region, particularly with regard to the elastic constant $C_{11}$. A poor reproducibility of the experimental data on $C_{11}$ for pure vanadium is a known problem, and it has been pointed out in a number of theoretical papers by other authors [21,38,39,40-42]. 


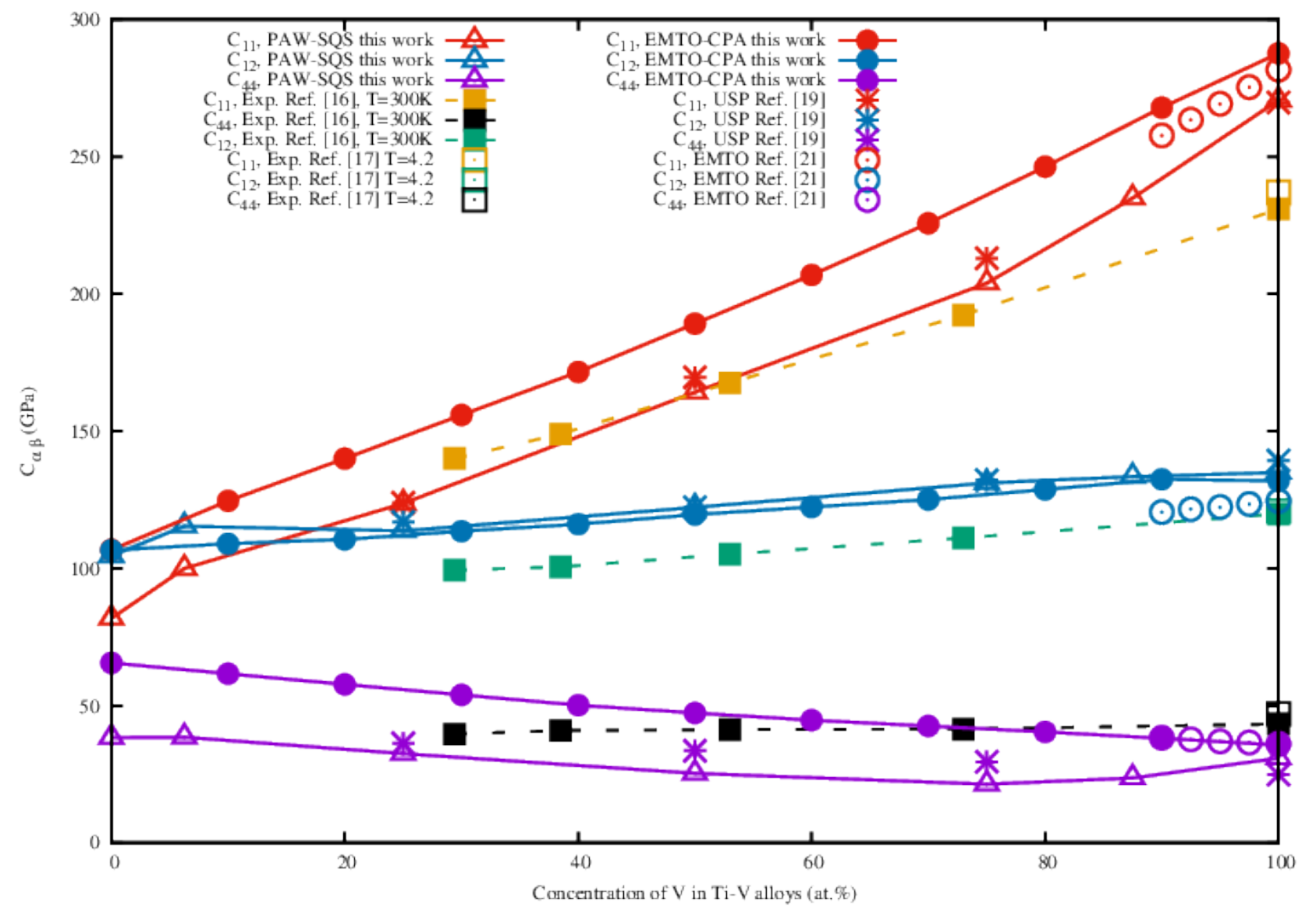

Fig. 1. Dependence of elastic constants $C_{11}, C_{12}, C_{44}$ on V concentration in bcc Ti-V alloy. Red symbols correspond to the calculated values of $C_{11}$. The calculated values of $C_{12}$ are shown as the blue symbols. Purple symbols are the calculated values of $C_{44}$. The gold dashed lines with filled square symbols denote the experimental values of $C_{11}$ Ref. [16], the experimental values of $C_{11}$ are shown as the gold empty square symbols (Ref. [17]). Green symbols correspond to the experimental values of $C_{12}$ (Refs. [16-17]). Black symbols are experimental values of $C_{44}$ from Refs. [16-17]. Solid lines with filled circles are the data obtained in this work by the EMTO-CPA method. The triangular symbols are the data obtained in this work by the PAW-SQS method. The values of Ref. [19] are marked with the asterisks symbols. The values of EMTO calculations from Ref. [21] are designated by the empty circular symbols.

Let us now discuss the elastic modulus $C_{44}$. The experimental data measured for bcc Ti-V alloys indicate that the value of $C_{44}$ is practically independent of the concentration and lies in the range from 40 to $43 \mathrm{GPa}$. It should be noted that the elastic constant $C_{44}$ obtained in the EMTOCPA calculations is monotonously decreasing with increasing $\mathrm{V}$ concentration. The curve obtained by the PAW-SQS method is not monotonous with a shallow minimum at 75 at. \% of V. The values obtained by the PAW-SQS method vary from 39 to $21 \mathrm{GPa}$ at vanadium concentrations from 0 to $100 \%$.

Considering results shown in Fig. 1, we did not observe, quite unexpectedly, a strong dependence of the elastic constants on the degree of chemical order in the system: our results for solid solutions are in very good agreement with those calculated in Ref. [19] for the ordered Ti-V alloys. However, we believe that this observation is specific for this particular system. For example, a strong influence of the degree of chemical order on the elastic moduli of $\operatorname{Ti}_{0.5} \mathrm{Al}_{0.5} \mathrm{~N}$ alloy was reported in Ref. [28]. 


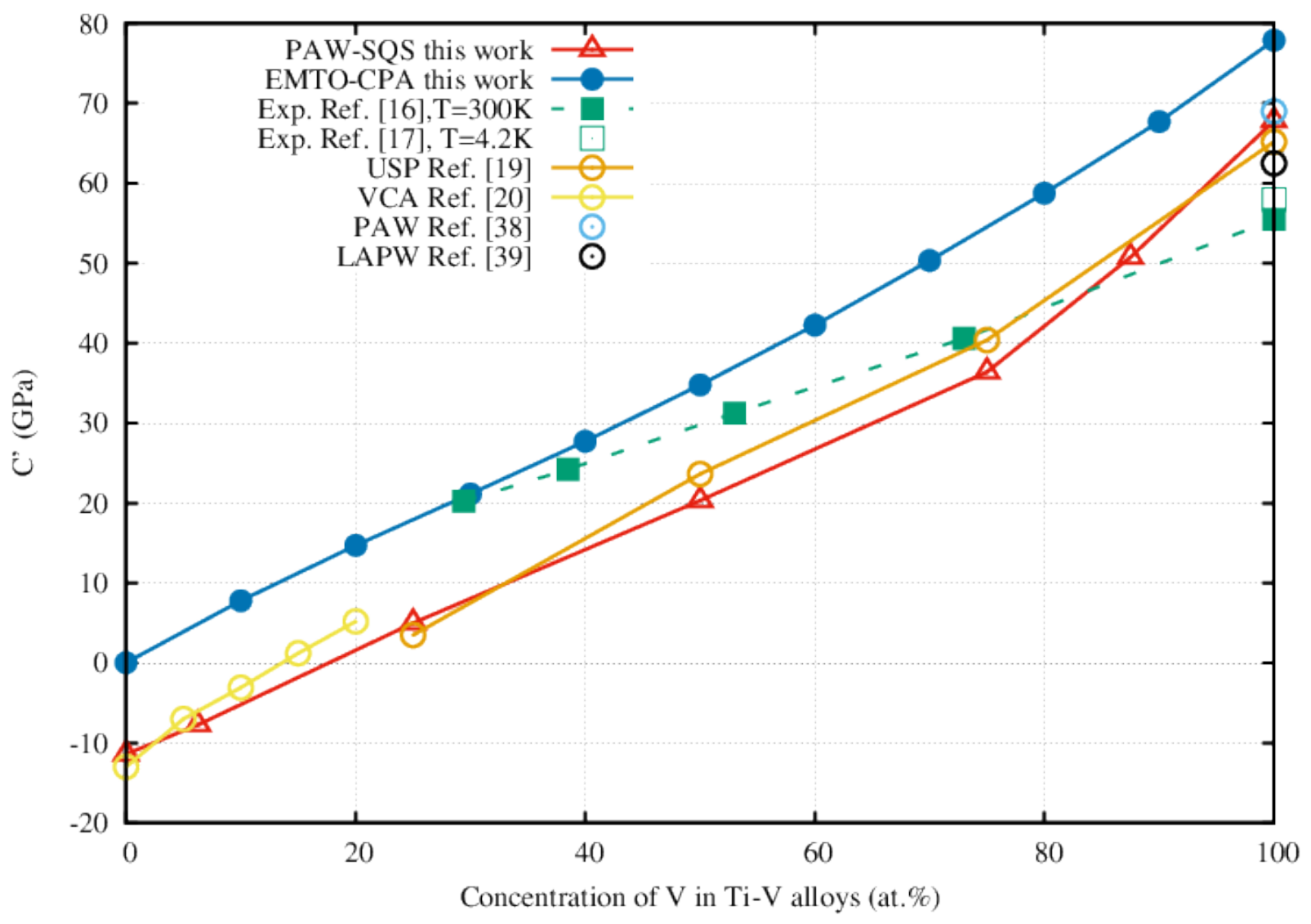

Fig. 2. Dependence of the elastic constant $C^{\prime}$ on $\mathrm{V}$ concentration. Red triangular symbols denote the values obtained by the PAW-SQS method in this work. Blue filled circles are the values obtained by us with the EMTO-CPA method. The green dashed lines with filled square symbols correspond the experimental data from Ref. [16]. The experimental data from Ref. [17] are designated by green empty square symbols. Gold color shows results of Ref. [19]. The values of VCA calculations reported in Ref. [20] are marked with the yellow symbols. Blue empty circles show PAW values of Ref. [38]. The LAPW values of Ref. [39] are indicated by the black symbols.

Figure 2 shows the dependence of the elastic constant $C^{\prime}$ on the concentration $\mathrm{V}$ in Ti-V alloys. Elastic constant $C^{\prime}$ obtained by the both methods increases monotonously with increasing vanadium concentration. The concentration dependence obtained by the PAW-SQS method is weakly nonlinear. Once again, there is a strong reason to believe that this is a numerical rather than physical effect, as both, the EMTO-CPA method and experiment indicate close to linear concentration dependence of $C^{\prime}$. In fact, a softening of this elastic constant can be used to characterize the mechanical stability of an alloy. Here one sees that $C^{\prime}$ becomes harder with increasing vanadium concentration, indicating the increasing mechanical stability of the system. One can also see that $C^{\prime}$ obtained by using the EMTO-CPA and PAW-SQS methods are in a reasonable agreement with the experimental values and with each other in the stability region of the solid solution.

On the other hand, there is an important qualitative difference between the results of PAW-SQS and EMTO-CPA calculations. According to the former, the $\beta$-phase of Ti becomes mechanically unstable at $\mathrm{V}$ concentrations below $\sim 20$ at. \%, where $C^{\prime}$ becomes negative. This is 
in good agreement with the experimental data, as well as with earlier theoretical studies $[16,20,43]$. On the contrary, the mechanical instability of the alloys in Ti-rich region is not reproduced by the EMTO-CPA method. Indeed, $C^{\prime}$ calculated by this technique is above 0 even at zero vanadium concentration. One therefore concludes that in critical regions of peculiarities associated with elastic constants the EMTO-CPA results must be verified with more accurate calculations.

Figure 3 shows the Young's modulus $E$, the bulk modulus $B$, the shear modulus $G$, and the Cauchy pressure $P_{c}$ as a function of the concentration $\mathrm{V}$ in the Ti-V alloy. One can see that $B$ and $P_{c}$ obtained by the PAW-SQS and EMTO-CPA methods increase almost linearly with increasing vanadium concentration. On the contrary, the dependences of $G$ and $E$ are strongly nonlinear. In addition, it should be noted that upon the approach to the area of mechanical instability the results obtained by the PAW-SQS method predict a sharp decrease of the Young's modulus. This fact can be important for a design of new alloys, e.g. for biomedical applications. In this case, the materials with relatively low values of Young's moduli close to the values of human tissue (10-60 GPa) are needed to ensure biomechanical compatibility and uniform load distribution, avoiding bone degradation.
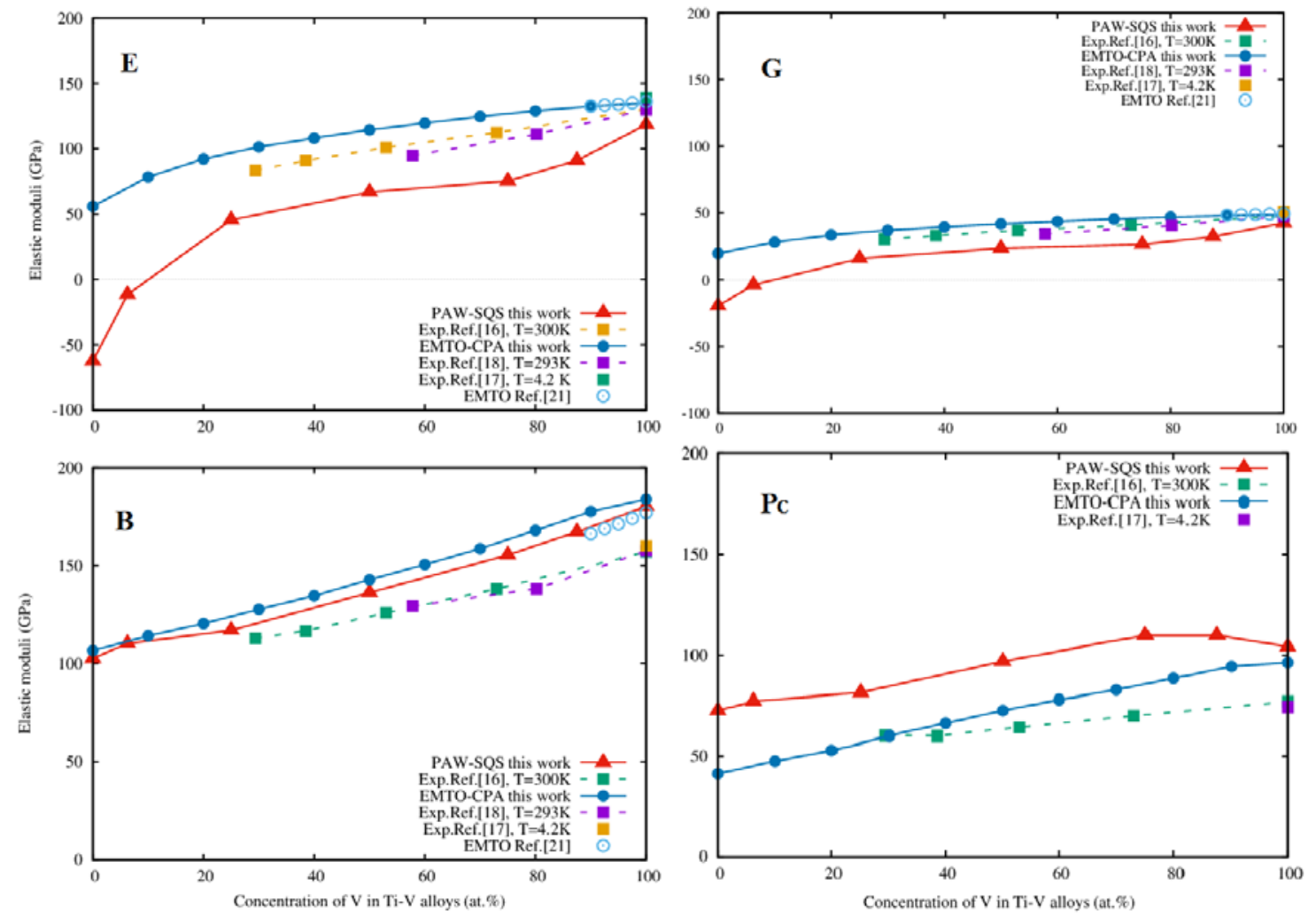

Fig. 3. Dependence of Young's modulus $E$, bulk modulus $B$, shear modulus $G$, and Cauchy pressure $P_{c}$ on $\mathrm{V}$ concentration in the bcc Ti-V alloy. The experimental data are shown as dashed lines of violet [16], green [18] and black [17] colors. The data obtained by the PAW-SQS method in this paper are denoted by red triangles. The data obtained by the EMTO-CPA method are marked with blue filled circles. Gold stars display the values of EMTO-CPA calculations from Ref. [21]. 
Currently the search for biocompatible materials is very active [44-45]. Though vanadium is not used for fabrication of biomedical implants because of its toxicity, alloys with low elastic moduli are needed as materials for stents and other biomechanical devices, where the materials with high elastic moduli would harm the human tissues. Usually such devices have coatings with special functions that in particular protect humans from toxic content. Besides, the materials with low elastic moduli could be useful for sensor systems, such as motion detectors and shock absorbers that provide damping of vibrations. Therefore, the results of the Young's modulus calculation for Ti-V alloys are of direct concentration behavior of the Young's modulus, which is difficult to predict based on either linear interpolations between values for the pure elements or by extrapolation of values obtained in the mechanically stable regions. This makes it possible to search for the optimal content of the alloying component, which brings the alloy to a vicinity of a mechanical instability, providing both mechanical stability and a desired value of the Young's modulus.

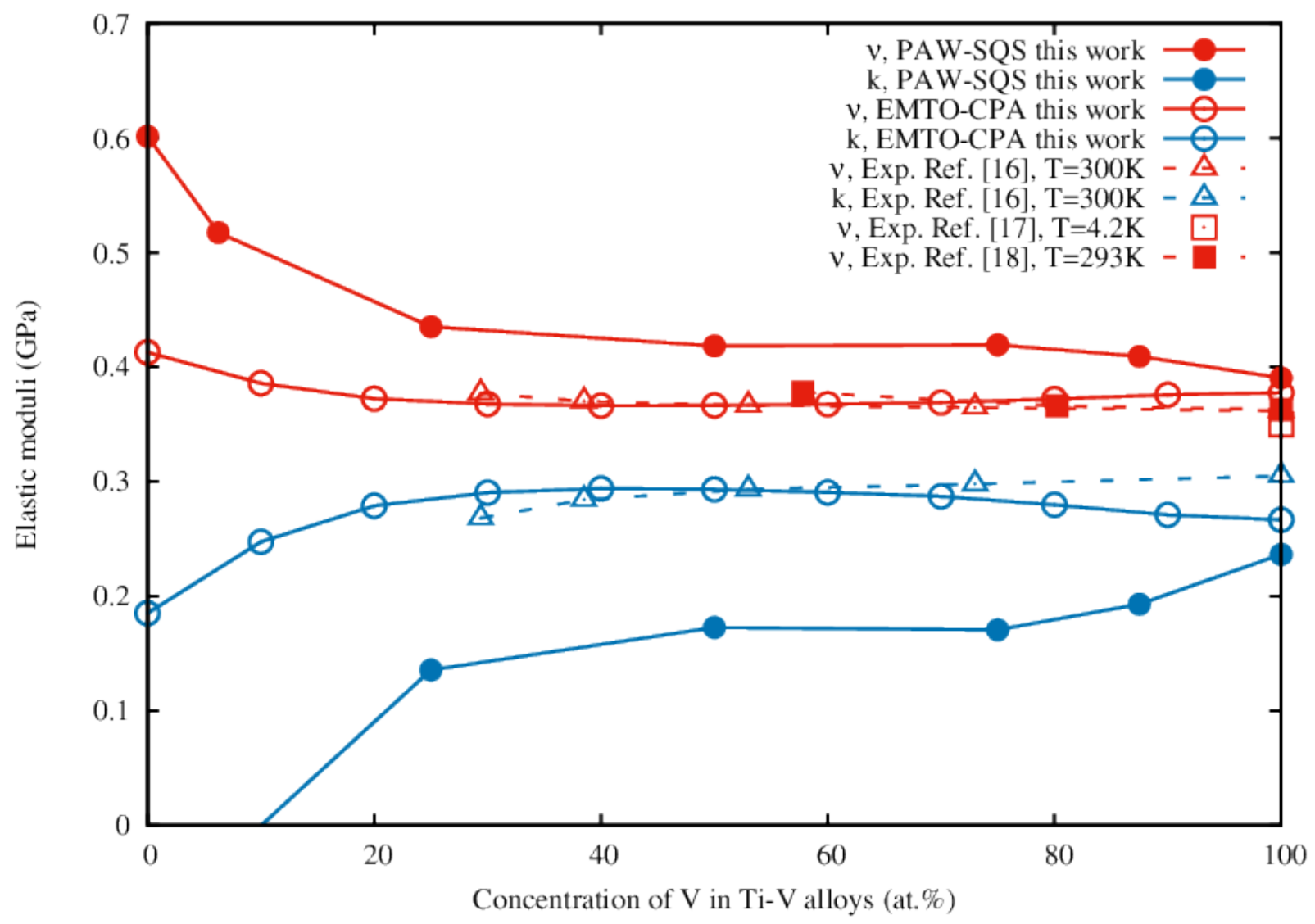

Fig. 4. Dependence of the Pugh's coefficient $k$ and the Poisson coefficient $v$ on Vanadium concentration in the bcc Ti-V alloy. Red symbols denote values of the Poisson's ratio. The blue symbols show the Pugh's coefficient values. Dashed lines with triangles and square symbols denote the experimental data. Filled circles show the data obtained by the PAW-SQS method in this paper. The data obtained by the EMTO-CPA method are shown as open circles.

Figure 4 shows the dependences of the Pugh's coefficient $k$ and the Poisson coefficient $v$ on the concentration $\mathrm{V}$ in the Ti-V alloy. The dependence of the coefficients on $\mathrm{V}$ concentration is quite weak in the region of mechanical stability of the bcc alloys, in agreement with experiment. On the other hand, it becomes quite strong for mechanically unstable alloys. 
The investigation of the concentration dependences of the $G / B$ ratio and the Cauchy pressure $P_{C}$ of the bcc Ti-V alloys carried out by using the phenomenological correlations between the ductility and the elastic constants indicates a possible slight increase in ductility with increasing vanadium content. The monotonously increasing values of $P_{c}$ suggest that the fraction of the metallic component of the interatomic bond increases with the addition of vanadium $\left(\mathrm{C}_{12}>\mathrm{C}_{44}\right)$. The analysis of the density of electronic states (DOS) (Figure 5) shows that strengthening of metallic bonding is provided, mainly, by the increasing occupation of $t_{2 g}$ and $e_{g}$ orbitals by $d$-electrons, which leads to a decrease of directional electron charge density distribution that becomes more spherical. It is seen from Fig. 5 that for the considered alloys consisting of $\mathrm{Ti}$ and $\mathrm{V}$ - neighboring elements in the periodic table - the rigid-band approximation works well. In fact, when vanadium is added, the number of valence electrons increases, and the Fermi level shifts toward the higher energy practically without changing the shape of the band. The main deviation from the approximation is an increase in the peak height located in the interval $-0.2--0.1$ Ry and formed predominantly by the states of $e_{g}$ symmetry. Since $e_{g}$-orbitals in bcc-type structures provide an atomic bond between the second neighbors, this effect leads to an increase in the elastic constant $C_{11}$ and, as a result, to the mechanical stabilization of the alloy. It should be noted, that the applicability of the rigid-band model is reflected in the good agreement of our PAW-SQS results and the data obtained by using the virtual crystal approximation (VCA) in Ref. [20] for the elastic constant $C^{\prime}$ (Fig. 2).

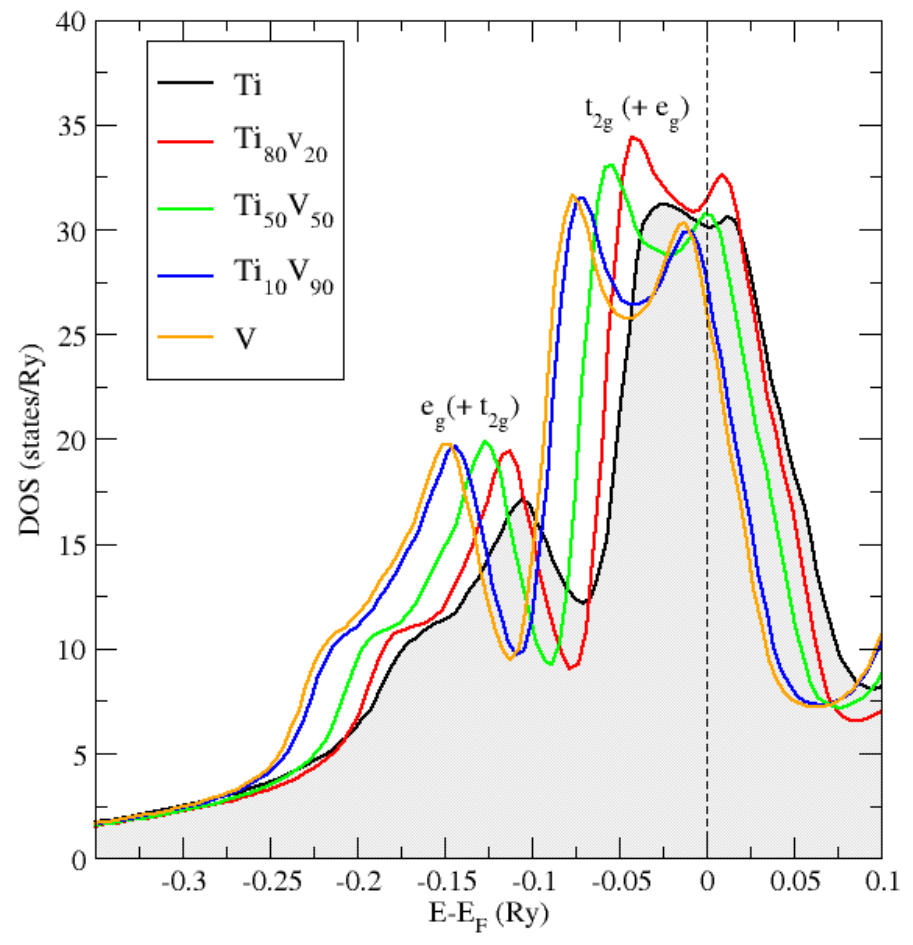

Fig. 5. Calculated total density of states of Ti-V alloys.

\section{Conclusions}

The elastic properties of the bcc Ti-V alloys were investigated from first-principles in the entire range of concentrations using the PAW and the EMTO methods. The substitutional disorder in the two methods was modeled using special quasi-random structure technique and the coherent 
potential approximation, respectively. The influence of $\mathrm{V}$ addition on the mechanical stability of the bcc crystal lattice for the alloys was studied. We have demonstrated that the PAW-SQS method reproduces the mechanical instability of Ti-rich alloys and therefore allows not only to obtain predictions of the values of elastic moduli in mechanically stable phases, but also to estimate the boundaries of the stability region. In the region of mechanical stability of bcc Ti-V alloys, the EMTO-CPA method predicts the elastic moduli in agreement with experimental data and more accurate theoretical methods. It can be used for an efficient study of concentration dependencies of elastic constants in multicomponent crystalline systems, since this method requires substantially less computational resources. The values of the elastic constants can be refined for alloys of particular interest with, e.g. PAW-SQS calculations, minimizing the total resources needed for the modeling. In using the latter methodology, particular care must be taken to ensure convergence of supercell calculations for a precise description of the concentration dependences of the elastic constants. The described computational strategy simplifies the process of high-throughput calculating the elastic constants for disordered alloys needed, e.g. for the next generation of data-bases of materials parameters.

The results obtained in this study indicate an increase of the mechanical stability of Ti$\mathrm{V}$ alloys with increasing vanadium content. It is shown that the $\beta$ phase of Ti stabilizes when vanadium content approaches 20 at. \% . Our calculations suggest new opportunities for solving

technologically relevant problems. For example, we have demonstrated that in the region of mechanical instability a significant reduction of the elastic moduli $E$ and $G$ are observed. Thus, it is possible to design materials with low values of, for instance, the Young's modulus, by optimizing the alloy composition in such a way as to be as close as possible to the mechanical instability, still remaining within the region of mechanically stable alloy compositions. Alloys with low values of the Young's modulus ( $\sim 50 \mathrm{GPa})$ are of high interest for, e.g. biomedical applications. Though $\mathrm{V}$ is not biocompatible, the strategy proposed above appears to be quite general, and can be used for a design of Ti-based alloys with biocompatible elements.

\section{Acknowledgements}

We are grateful for the support provided by the Grant of the Ministry of Education and Science of the Russian Federation (Grant No. 14.Y26.31.0005) and the Swedish Government Strategic Research Area in Materials Science on Functional Materials at Linköping University (Faculty Grant SFO-Mat-LiU No 2009 00971). The calculations were performed on resources provided by the Swedish National Infrastructure for Computing (SNIC) at the National Supercomputer Centre (NSC) and the supercomputer cluster provided by the Materials Modeling and Development Laboratory at NUST “MISIS”.

\section{References}

1. M. de Jong, W. Chen, R. Notestine, K. Persson, G. Ceder, A. Jain, M. Asta, and A. Gamst, A Statistical Learning Framework for Materials Science: Application to Elastic Moduli of knary, Inorganic Polycrystalline Compounds (2016) Scientific Reports, 6, 34256, https://10.1038/srep34256 
2. A. Zunger, S.H. Wei, L.G. Ferreira, and J.E. Bernard, Special quasirandom structures, Physical Review Letters, (1990) 65 (3), 353-356, https://10.1103/PhysRevLett.65.353

3. D.B. Laks, L.G. Ferreira, S. Froyen, and A. Zunger, Efficient cluster expansion for substitutional systems, Physical Review B (1992) 46 (19), 12587-12605, https://10.1103/PhysRevB.46.12587

4. L. Vitos, Computational Quantum Mechanics for Materials Engineers, Springer London, London, 2007.

5. P.E. Blöchl, Projector augmented-wave method, Physical Review B (1994) 50 (24), 1795317979, https://10.1103/PhysRevB.50.17953

6. L. Vitos, I.A. Abrikosov, and B. Johansson, Anisotropic Lattice Distortions in Random Alloys from First-Principles Theory, Phys. Rev. Lett. (2001) 87, 156401, https://10.1103/PhysRevLett.87.156401

7. R. Chinnappan, B.K. Panigrahi, and A. van de Walle, First-principles study of phase equilibrium in Ti-V, Ti-Nb, and Ti-Ta alloys, Calphad (2016) 54, 125-133, https://10.1016/j.calphad.2016.07.001

8. M. Sluiter and P.E.A. Turchi, Phase stability in Ti-V and Ti-Cr alloys: A theoretical investigation , Phys. Rev. B (1991) 43, 12251-12266, https://10.1103/PhysRevB.43.12251

9. A. Kostov and D. Zivković, Thermodynamic analysis of alloys Ti-Al, Ti-V, Al-V and Ti-AlV，J. Alloys Compd. (2007) 460, 164-171, https://10.1016/j.jallcom.2007.05.059

10. L.-J. Gui, Y.-L. Liu, W.-T. Wang, Y. Wei, Y. Zhang, G.-H. Lu, and J.-E. Yao, Behaviors of alloying element titanium in vanadium: From energetics to tensile/shear deformation, Comput. Mater. Sci. (2013) 77, 348-354, https://10.1016/j.commatsci.2013.04.064

11. S. Das, Physical Aspects of Process Control in Selective Laser Sintering of Metals , Adv. Eng. Mater. (2003) 5, 701, https://10.1002/adem.200310099

12. S. Suwarno, J.K. Solberg, J.P. Maehlen, B. Krogh, and V.A. Yartys, The effects of rapid solidification on microstructure and hydrogen sorption properties of binary BCC Ti-V alloys, J. Alloys Compd. (2014) 582, 540, https://10.1016/j.jallcom.2013.08.077

13. T. Ozaki, H. Matsumoto, S. Watanabe, and S. Hanada, Beta ti alloys with low young's modulus, Mater. Trans. (2004) 45, 2776.

14. S. Ehtemam-Haghighi, K.G. Prashanth, H. Attar, A.K. Chaubey, G.H. Cao, L.C. Zhang, Evaluation of mechanical and wear properties of TixNb7Fe alloys designed for biomedical applications, In Materials \& Design (2016), 592-599, https://doi.org/10.1016/j.matdes.2016.09.029

15. H. Miao and W.G. Wang, Mechanisms of improving the cyclic stability of V-Ti-based hydrogen storage electrode alloys , J. Alloys Compd. (2010) 508, 592-598,

https://10.1016/j.jallcom.2010.08.132

16. K.W. Katahara, M.H. Manghnani, and E.S. Fisher, Pressure derivatives of the elastic moduli of BCC Ti-V-Cr, Nb-Mo and Ta-W alloys, J. Phys. F Met. Phys. (1979) 9, 773, https://10.1088/0305-4608/9/5/006

17. D.I. Bolef, R.E. Smith, and J.G. Miller, Elastic properties of vanadium. I. Temperature dependence of the elastic constants and the thermal expansion, Phys. Rev. B (1971) 3, 41004108, https://10.1103/PhysRevB.3.4100

18. J.T. Lenkkeri and E.E. Lahteenkorva, An investigation of elastic moduli of vanadiumchromium alloys, J. Phys. F Met. Phys. (1978) 8, 1643-1651, https://10.1088/03054608/8/8/006

19. H. Ikehata, N. Nagasako, T. Furuta, A. Fukumoto, K. Miwa, and T. Saito,First-principles calculations for development of low elastic modulus Ti alloys, Physical Review B (2004) 70 (17), 174113, https://10.1103/PhysRevB.70.174113 
20. P. Söderlind, A. Landa, L.H. Yang, and A.M. Teweldeberhan, First-principles phase stability in the Ti-V alloy system, J. Alloys Compd. (2013) 581, 856, https://10.1016/j.jallcom.2013.07.138

21. X. Li, H. Zhang, S. Lu, W. Li, J. Zhao, B. Johansson, and L. Vitos, Elastic properties of vanadium-based alloys from first-principles theory, Phys. Rev. B (2012) 86, 14105, https://10.1103/PhysRevB.86.014105

22. R. Sahara, S. Emura, and K. Tsuchiya, Theoretical investigation of effect of alloying elements on phase stability in body-centered cubic Ti-X alloys (X = V, Cr, Fe, Co, Nb, and Mo), J. Alloys Compd. (2015) 634, 193, https://10.1016/j.jallcom.2015.02.005

23. G. Kresse and J. Furthmüller, Efficiency of ab-initio total energy calculations for metals and semiconductors using a plane-wave basis set, Comput. Mater. Sci. (1996) 6, 15, https://10.1016/0927-0256(96)00008-0

24. G. Kresse and J. Furthmüller, Efficient iterative schemes for ab initio total-energy calculations using a plane-wave basis set, Phys. Rev. B (1996) 54, 11169-11186, https://doi.org/10.1103/PhysRevB.54.11169

25. J.P. Perdew, K. Burke, and M. Ernzerhof, Generalized gradient approximation made simple, Phys. Rev. Lett. (1996) 77, 3865-3868, DOI: 10.1103/PhysRevLett.77.3865

26. J. Koll'ar, L. Vitos, H. L. Skriver, The Uses of the LMTO Method, Lecture Notes in Physics, in: H. Dreyss'e (Eds.), Electronic Structure and Physical Properties of Solids, Springer, Berlin (2000), p. 85.

27. P. Steneteg, O. Hellman, O.Y. Vekilova, N. Shulumba, F. Tasnádi, and I.A. Abrikosov, Temperature dependence of TiN elastic constants from ab initio molecular dynamics simulations, Phys. Rev. B (2013) 87, 94114, https://10.1103/PhysRevB.87.094114

28. F. Tasnádi, M. Odén, and I.A. Abrikosov, Ab initio elastic tensor of cubic Ti $0.5 \mathrm{Al} 0.5 \mathrm{~N}$ alloys: Dependence of elastic constants on size and shape of the supercell model and their convergence, Phys. Rev. B (2012) 85, 144112, https://10.1103/PhysRevB.85.144112

29. M. Moakher and A. N. Norris, The Closest Elastic Tensor of Arbitrary Symmetry to an Elasticity Tensor of Lower Symmetry, J. Elast. (2006) 85, 215-263, https://link.springer.com/article/10.1007\%2Fs10659-006-9082-0

30. W. Voigt, Lehrbuch der Kristallphysik, Taubner, Leipzig (1928)

31. A. Reuss, Berechnung der Fließgrenze von Mischkristallen auf Grund der Plastizitätsbedingung für Einkristalle , ZAMM - J. Appl. Math. Mech. (1929) 9, 49-58, https://10.1002/zamm.19290090104

32. R. Hill, The elastic behaviour of a crystalline aggregate, Proc. Phys. Soc. Sect. A (1952) 65, 349, https://10.1088/0370-1298/65/5/307

33. G. Grimvall, Thermophysical Properties of Materials, 1st ed. (1999) Elsevier, New York

34. C. Asker, L. Vitos, and I. A. Abrikosov, Elastic constants and anisotropy in FeNi alloys at high pressures from first-principles calculations, Phys. Rev. B (2009) 79, 214112, https://doi.org/10.1103/PhysRevB.79.214112

35. D.G. Pettifor, Theoretical predictions of structure and related properties of intermetallics, Mater. Sci. Technol. (1992) 8, DOI: 10.1179/mst.1992.8.4.345

36. S.F. Pugh, Relations between the elastic moduli and the plastic properties of polycrystalline pure metals , Philos. Mag. J. Sci. (1954) 45, 823-843,

http://10.1080/14786440808520496

37. P.A.T. Olsson, M. Mrovec, and M. Kroon, First principles characterisation of brittle transgranular fracture of titanium hydrides, Acta Mater. (2016) 118, 362-373 http://10.1016/j.actamat.2016.07.037 
38. X. Li, C. Zhang, J. Zhao, and B. Johansson, Mechanical properties and defective effects of bcc V-4Cr-4Ti and V-5Cr-5Ti alloys by first-principles simulations, Comput. Mater. Sci. (2011) 50, 2727-2731, http://10.1016/j.commatsci.2011.04.027

39. M.J. Mehl and D.A. Papaconstantopoulos, Applications of a tight-binding total-energy method for transition and noble metals: Elastic constants, vacancies, and surfaces of monatomic metals, Phys. Rev. B (1996) 54, 4519-4530, https://doi.org/10.1103/PhysRevB.54.4519

40. M. Jahnátek, M. Krajčí, and J. Hafner, Interatomic bonding, elastic properties, and ideal strength of transition metal aluminides: A case study for Al3(V,Ti), Phys. Rev. B (2005) 71, 24101, https://doi.org/10.1103/PhysRevB.71.024101

41. L. Koči, Y. Ma, A.R. Oganov, P. Souvatzis, and R. Ahuja, Elasticity of the superconducting metals V, Nb, Ta, Mo, and W at high pressure, Phys. Rev. B (2008) 77, 214101, https://doi.org/10.1103/PhysRevB.77.214101

42. X. Zhou, D. Gall, and S. V. Khare, First-principles phase diagram calculations for the rocksalt-structure quasibinary systems TiN-ZrN, TiN-HfN and ZrN-HfNJ, Alloys Comd. (2014) 595, 80 , https://doi.org/10.1088/0953-8984/29/3/035401

43. A.V. Dobromyslov and V.A. Elkin, Phase and structural transformations in Ti-Ta alloys, Scr. Mater. (2001) 44, 905, https://doi.org/10.1134/S0031918X09050111

44. P.F. Santos, M. Niinomi, H. Liu, K. Cho, M. Nakai, A. Trenggono, S. Champagne, H. Hermawan, T. Narushima, Improvement of microstructure, mechanical and corrosion properties of biomedical Ti-Mn alloys by Mo addition, In Materials \& Design (2016), 414424, https://doi.org/10.1016/j.matdes.2016.07.115

45. P. S. Nnamchi, First principles studies on structural, elastic and electronic properties of new TiMoNbZr alloys for biomedical applications, In Materials \& Design (2016), 60-67, https://doi.org/10.1016/j.matdes.2016.06.066 


\title{
AB INITIO CALCULATIONS OF ELASTIC PROPERTIES OF ALLOYS WITH MECHANICAL INSTABILITY: APPLICATION TO BCC Ti-V alloys.
}

\author{
N. V. Skripnyak ${ }^{1}$, A. V. Ponomareva ${ }^{2}$, M. P. Belov², I. A. Abrikosov" ${ }^{1, *}$ \\ ${ }^{1}$ Department of Physics, Chemistry and Biology, Linköping University, Linköping, SE-581 83, Sweden \\ ${ }^{2}$ Materials Modeling and Development Laboratory, National University of Science and Technology “MISIS", \\ Moscow, 119049, Russia
}

\section{Supporting information}

\section{Optimization of parameters for calculations of elastic properties of $\mathrm{Ti}-\mathrm{V}$ alloys.}

A set of tests was carried out to determine the optimal calculation parameters. Let us start with the pure alloy components, which are modeled using the same size supercells as for the alloys to minimize the numerical errors. First, a test was performed on the k-points convergence. For pure vanadium, an additional test for a simple cubic cell with $2 \mathrm{~V}$ atoms was performed to find elastic constants that are fully converged in terms of k-points. The results of this study are shown in Table S1.

In order to determine the elastic constants $\mathrm{C}^{\prime}$ and $\mathrm{C} 44$ of $\mathrm{Ti}-\mathrm{V}$ alloys we applied volumeconserving orthorhombic (1) and monoclinic (2) distortions as well as volume-non-conservation deformation matrix (3).

$$
\begin{aligned}
1+\varepsilon_{1}(\eta) & =\left(\begin{array}{ccc}
1+\eta & 0 & 0 \\
0 & 1-\eta & 0 \\
0 & 0 & \frac{1}{1-\eta^{2}}
\end{array}\right) \\
1+\varepsilon_{2}(\eta) & =\left(\begin{array}{ccc}
1 & \eta & 0 \\
\eta & 1 & 0 \\
0 & 0 & \frac{1}{1-\eta^{2}}
\end{array}\right) \\
1+\varepsilon_{1}(\eta) & =\left(\begin{array}{ccc}
1+\eta & \frac{\eta}{2} & 0 \\
\frac{\eta}{2} & 1 & 0 \\
0 & 0 & 1
\end{array}\right)
\end{aligned}
$$

Table SI. Dependence of elastic constants (in GPa) on the number of k-points used for the Brillouin zone integration in PAW-SQS calculations for pure titanium and pure vanadium.

\begin{tabular}{|c|c|c|c|c|c|c|}
\hline & \multicolumn{2}{|c|}{ k-points } & $\mathrm{C}_{11}$ & $\mathrm{C}_{12}$ & $\mathrm{C}_{44}$ & $C^{\prime}$ \\
\hline \multirow{3}{*}{$\begin{array}{l}\text { Ti (128 } \\
\text { supercell) }\end{array}$} & 1 & 111 & 97.6 & 102.1 & 9.2 & -2.2 \\
\hline & 8 & 222 & 86.7 & 110.5 & 38.4 & -11.9 \\
\hline & 14 & 333 & 86.8 & 110.9 & 39.5 & -12.1 \\
\hline V (128 & 1 & 111 & 366.0 & 69.1 & 27.4 & 148.4 \\
\hline
\end{tabular}
Calculations were carried out for 128 atom Ti supercell, 128 atom V supercell and for simple cubic cell with $2 \mathrm{~V}$ atoms with the bcc underlying lattice. 


\begin{tabular}{|c|c|c|c|c|c|c|}
\hline \multirow[t]{2}{*}{ Supercell) } & 8 & 222 & 242.3 & 153.8 & 23.3 & 44.2 \\
\hline & 14 & 333 & 270.5 & 134.9 & 30.9 & 67.8 \\
\hline \multirow{15}{*}{$\begin{array}{l}\text { V (simple } \\
\text { cubic cell } \\
\text { with } 2 \\
\text { atoms) }\end{array}$} & 260 & $8 \times 8 x 8$ & 242.6 & 157.2 & 23.2 & 42.7 \\
\hline & 365 & $9 \times 9 \times 9$ & 245.7 & 154.8 & - & 45.4 \\
\hline & 504 & $10 \times 10 \times 10$ & 274.2 & 141.4 & 15.9 & 66.4 \\
\hline & 666 & $11 \times 11 \times 11$ & 268.5 & 145.6 & 23.8 & 61.4 \\
\hline & 868 & $12 \times 12 \times 12$ & 276.8 & 137.7 & 28.0 & 69.5 \\
\hline & 1099 & $13 \times 13 \times 13$ & 271.9 & 141.2 & 22.8 & 65.3 \\
\hline & 1376 & $14 \times 14 \times 14$ & 272.6 & 139.8 & 26.6 & 66.4 \\
\hline & 1688 & $15 \times 15 \times 15$ & 273.3 & 140.4 & 25.9 & 66.5 \\
\hline & 2052 & $16 \times 16 \times 16$ & 267.5 & 146.0 & 21.4 & 60.7 \\
\hline & 2457 & $17 \times 17 \times 17$ & 270.3 & 143.9 & 22.2 & 63.2 \\
\hline & 2920 & $18 \times 18 \times 18$ & 272.3 & 140.5 & 24.8 & 65.9 \\
\hline & 3430 & $19 \times 19 \times 19$ & 271.2 & 141.2 & 24.5 & 65.0 \\
\hline & 4004 & $20 \times 20 \times 20$ & 271.1 & 143.2 & 23.2 & 63.9 \\
\hline & 4631 & $21 \times 21 \times 21$ & 271.0 & 143.1 & 23.5 & 63.9 \\
\hline & 5328 & $22 \times 22 \times 22$ & 271.9 & 142.3 & 24.0 & 64.8 \\
\hline
\end{tabular}

As can be seen from the Table SI, different sets of parameters are needed to obtain convergence for different elements. Indeed, for 128 atom supercell of pure titanium a grid of 2x2x 2 k-points is enough, but vanadium behaves differently. Comparing the test calculations carried out for 128 atom supercell of pure $\mathrm{V}$ with fully converged results obtained for the simple cubic cell with 2 atoms, one sees that for $\mathrm{V}$ a grid of at least $3 \times 3 \times 3$ k-points is required to converge elastic constants $C_{11}$ and $C^{\prime}$. The modulus $C_{12}$ is converged with $\sim 6 \%$ at this k-points grid. Unfortunately, even denser k-point mesh seems to be needed to converge the elastic modulus $C_{44}$. We note, however, that its value is very low, explaining larger relative error. Giving this fact, and considering too high computational costs of further increase of the k-point density in our PAW-SQS calculations, we limit the grid of k-points to 3x3x3 in this work.

Further, a series of tests using various calculation parameters were performed for an alloy with vanadium concentration 75 at. \%. The calculations were carried out with various SQS with and without relaxation of the local atomic positions in the SQS. The deformation matrices that do not conserve the volume of the cell, and also the orthorhombic and monoclinic matrices preserving the volume were used. The integration of the Brillouin zone was performed using two sets of k-points, $2 \times 2 \times 2$ and $3 \times 3 \times 3$.

The following enumeration of the calculations will be used in the following discussion. In calculation №1 a deformation matrix given in Eq. (3) was employed. Remember that the deformation matrix does not conserve the volume, and that all the obtained values are averaged over 3 independent directions. Calculation №2 was carried out in the same way as calculation №1. However, a different SQS (with the same composition) was used to verify the quality of our SQS structures in calculations of elastic moduli. In the both calculations the alloy components occupied the ideal positions of the bcc underlying lattice, that is the effect of the local lattice relaxations was neglected. Calculation №3 was carried out by analogy with the calculation №1. However, here local relaxations were carried out on undeformed SQS-1, and then the local positions of the atoms in the SQS were not re-optimized in the calculations of the supercells distorted according to Eq. (3). In calculation №4, on the other hand, the local relaxations were 
performed for SQS-1 at each degree of deformation. Calculation №5 was performed using monoclinic and orthorhombic deformation matrices (1) - (2) and an ideal SQS structure. Again, all the calculated elastic moduli were averaged over 3 independent directions. Calculation №6 was carried out in the same way as calculation №1, but it used a different set of k-points. For comparison, we used the experimental data on the alloy with $\mathrm{V}$ concentration 73 at. \% [1]. The results of the study are presented in Table SII.

Table SII. Elastic constants of Ti- 75\% V (in GPa), calculated using the PAW-SQS method with different setups.

\begin{tabular}{|c|c|c|c|c|c|c|c|c|}
\hline $\begin{array}{l}\text { Calculat } \\
\text { ion }\end{array}$ & $\begin{array}{l}\text { Type of } \\
\text { structure }\end{array}$ & Relax & matrix & $\begin{array}{l}\text { Grid K- } \\
\text { points }\end{array}$ & $\mathrm{C}_{11}$ & $\mathrm{C}_{12}$ & $\mathrm{C}_{44}$ & $C^{\prime}$ \\
\hline № 1 & SQS1 & No & Eq. (3) & $2 \times 2 x 2$ & 222.5 & 124,5 & 26.8 & 49.0 \\
\hline № 2 & SQS2 & No & Eq. (3) & $2 \times 2 x 2$ & 222.7 & 123.5 & 27.0 & 49.6 \\
\hline № 3 & SQS1 & $\begin{array}{l}\text { Yes, for } \\
\text { initial } \\
\text { structure }\end{array}$ & Eq. (3) & $2 \times 2 \times 2$ & 229.2 & 125.1 & 34.1 & 52.0 \\
\hline № 4 & SQS1 & $\begin{array}{l}\text { Yes, for } \\
\text { each } \\
\text { structure } \\
\text { s for } \\
\text { each } \\
\text { deforma } \\
\text { tion }\end{array}$ & Eq. (3) & $2 \times 2 x 2$ & 219.1 & 122.8 & 25.2 & 48.1 \\
\hline № 5 & SQS1 & No & $\begin{array}{l}\text { Eq. (1- } \\
2)\end{array}$ & $2 \times 2 \times 2$ & & & 26.3 & 46.9 \\
\hline № 6 & SQS1 & No & Eq. (3) & 3x3x3 & 203.9 & 131.1 & 21.4 & 36.4 \\
\hline $\begin{array}{l}\text { Calculat } \\
\text { ion, Ref. } \\
\text { [2] }\end{array}$ & $\begin{array}{l}\text { Ordered } \\
\text { structure }\end{array}$ & & & & 213.0 & 132.2 & 29.6 & 40.4 \\
\hline
\end{tabular}




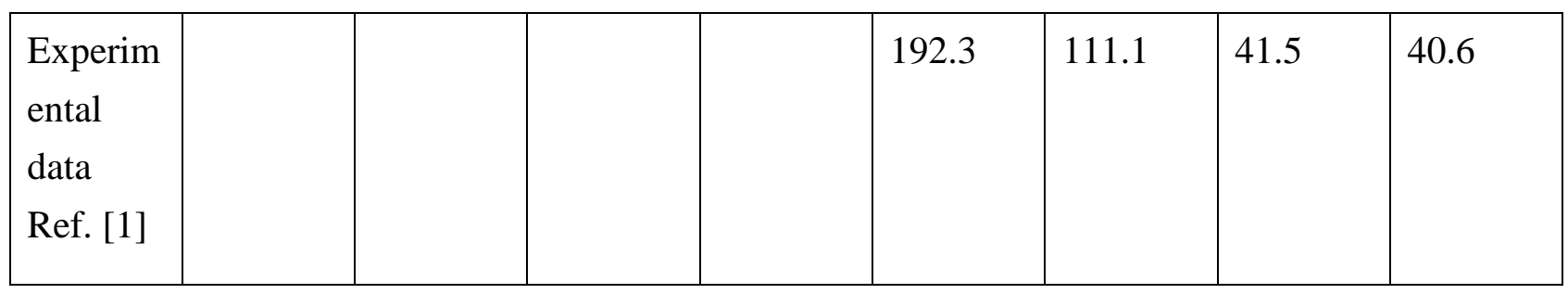

Table SII shows that the results of the calculations of $C_{11}$ and $C_{12}$ carried out with the different computational schemes differ within $5 \%$. In the case of $C_{44}$ and $C^{\prime}$, the absolute error does not exceed 5.2 GPa. It was found that the calculated values of the elastic moduli depend primarily on the set of k-points and do not depend on the type of SQS. The form of the matrix used in the calculations does not affect the result either. Importantly, we observed that conducting the procedure of ionic relaxations does not affect the calculated elastic constants. The observation is important for the use of the EMTO-CPA method which neglects the effect of the local lattice relaxations. Quite unexpectedly, we did not observe strong dependence of the elastic constants on the degree of chemical order in the system: our results are in very good agreement with those calculated in Ref. [2] for the ordered Ti-V alloys. However, we believe that this observation is accidental. For example, a strong influence of the degree of chemical order on the elastic moduli of $\mathrm{Ti}_{0.5} \mathrm{Al}_{0.5} \mathrm{~N}$ alloy was observed in Ref. [3].

\section{References}

1. K.W. Katahara, M.H. Manghnani, and E.S. Fisher, J. Phys. F Met. Phys. 9, 773 (1979).

2. R. Sahara, S. Emura, and K. Tsuchiya, J. Alloys Compd. 634, 193 (2015).

3. F. Tasnádi, M. Odén, and I.A. Abrikosov, Phys. Rev. B 85, 144112 (2012). 